\title{
COMBINING TERRESTRIAL SCANNED DATASETS WITH UAV POINT CLOUDS FOR MINING OPERATIONS
}

\author{
Megan Fotheringham and Dev Raj Paudyal \\ School of Civil Engineering and Surveying \\ University of Southern Queensland, Australia
}

Commission IV, WG IV/6

KEY WORDS: Unmanned Aerial Vehicles (UAV), Terrestrial Laser Scanning, Spatial Data Integration, Digital Surface Models, Point Cloud Datasets, Mining Operations

\begin{abstract}
:
Surveyors of open cut mining operations employ multiple data acquisition techniques such as the use of Unmanned Aerial Vehicles (UAV), Terrestrial Laser Scanning (TLS) and GNSS positioning for creating 3D surface models. Surveyors, mine planners and geologists are increasingly combining point cloud datasets to achieve more detailed surface models for the use of material reconciliation and volume calculations. Terrestrial Laser Scanning and UAV photogrammetry have enabled large, accurate and time effective data collection and increased computing capacity enables geospatial professionals to create 3D virtual surfaces, through merging UAV point clouds and TLS data combing with GNSS positioning. This research paper investigates the effects of combining data sets for creating 3D surface models from independent spatial data collection methods such as UAV, TLS and GNSS and assess their accuracy for the purpose of volume calculations in mining operation. 3D surface models provide important information for mining operations, planning of resources, material volumes calculation and financial calculations. A case study of two rehabilitation mine sites in Northern Victoria, Australia was selected for this study. Field data were collected using Terrestrial Laser Scanner and UAV. After each dataset was processed and filtered, the data were merged to create surface models. The accuracy of the combined model was assessed comparing height $(\mathrm{Z})$ values using a fishnet point grid of the surfaces. Volumes between surfaces were calculated, and a cost applied to the results based on the current bulk cubic meter (BCM) haulage rates. The outputs from this study will provide scientific contributions to civil and mining industries where the computation of stockpile values is required.
\end{abstract}

\section{INTRODUCTION}

\subsection{Background}

In open cut mining, conventional surveying method is used to monitor mineral production, high wall and ground stability, as well as pit configuration. However, this traditional surveying method is high risk to personal safety while surveying around surface mining equipment (SME) and can put surveyors in 'the line of fire' whilst also being time consuming. Mine surveyors are tasked with collecting daily surveys for operational requirements, ensuring accuracy of plans and construction set out, maintaining machine guidance equipment and plans, and performing high precision measurements in high pressure and unforgiving environments. One of the biggest tasks for mine surveyors are the end of month (EOM) data collection and reporting. End of month surveys provide a snapshot of the mining operations from the previous month and are used to create and update life-of-mine plans. One of the major functions for EOM surveys is the generation of surface 3D models used for planning and volumetric computation. Volumes are required to calculate the amount of material for extraction and for confirmation of materials moved throughout the month, these calculations are required for contractors' payments and estimates of profit and expenses.

Over the past decade, surveying in open cut mining operations has continued to change with technology, starting with the availability and accessibility of GNSS equipment, incorporating terrestrial laser scanning and now UAV photogrammetry. Data collection through photogrammetry has proven its worth for mining with a large portion of the data collection in open cut operations now achieved with the use of drones and UAVs.
With the ability to cover large amount areas in an efficient time, UAVs are allowing surveyors access to areas of mining sites that have previously been inaccessible due to terrain or safety concerns.

\subsection{Mineral oil extraction and the rehabilitation of land}

Mineral sands contain important mineral deposits known as heavy metals, these include minerals rich in titanium, zircon, rutile, ilmenite, and rare earth elements. In Australia, Victoria has extensive deposits of mineral sands including rutile, ilmenite, zircon, monazite, and rare earths. In 2011, Victoria had 21.8\% of Australia's Economic Demonstrated Resource (EDR) of ilmenite, $33.8 \%$ of its zircon and $42.3 \%$ of its rutile (Iluka Resources 2020).

Iluka resources have conducted open cut mining operations within the Murray Basin deposits in Victoria since 2009, and alongside the extraction of resources, rehabilitation of the disturbed landscape has been underway. In 2012-2013, Victoria's mineral sands production was 86,243 tonne of zircon, 140,842 tonnes of rutile and 67,067 tonnes of ilmenite at a total estimated net market value of $\$ 282.7$ million (Iluka Resources 2020).

The rehabilitation of land is important for the completion of mining operations, and is considered from early stages of development, with environmental surveys and studies completed alongside survey of pre mine land formations. The rehabilitation plan is submitted to the mining department, so companies are held accountable. The process for the rehabilitation of land mined commences with the collection of critical environmental data and the development of 
rehabilitation objectives before mining commences. 'Initial field surveys involve studies on current and proposed land use, topography, the condition of any infrastructure and flora and fauna populations. Any vegetation and infrastructure are removed before mining commences, while stockpiling of the surface layer and seed collection may also occur. This process varies depending on the post-mining objectives, such as restoration of land to agricultural use, native vegetation or commercial development (Iluka Resources 2020).

\subsection{Stockpile formation}

During open cut mining operations layers of soil are removed and stockpiled to access the ore reserves, there are generally three types of material striped to expose the ore body, topsoil this might include various grades with different nutrient values, subsoil, and overburden, with material being stockpiles for future rehabilitation works, 'The surface layer of the soil is the biologically active component, containing the bulk of the nutrient, organic matter, soil fauna and microbial activity that is essential for effective mine rehabilitation. In areas where the ore body is close to the surface, only the topsoil is stripped and stockpiled and the rest is mined (Victoria State Government, 2020). After the creation of stockpiles, surveyors would capture the surface data for volume calculations and future planning. This was usually done with a GNSS rover and manually collected by walking top and toe contours or continuous TOPO collection from a vehicle with the GNSS receiver mounted to the front of the vehicle.

\section{LITERATURE REVIEW}

\subsection{Data collection methods}

Within the last decade Terrestrial Laser Scanning (TLS) and Unmanned Aerial Vehicles (UAV) have increased in popularity amongst the surveying and spatial science communities with the promise of fuller data sets as the ability to analyse these large files with the rapid advancement in technology. The costs of buying new equipment such as UAVs and laser scanners have reduced and the benefits such as time and labour savings make them an attractive option, as discussed through Cook (2017); and Akturk (2018). This is especially so for high risk jobs such as mining and natural resource monitoring Javernick, et al. (2014); Tong,et al. (2015); and Szentpeteri,et al. (2016). UAVs are being used in place of LiDAR for smaller time sensitive acquisition, and with fast rates of technology turn over, multirotor systems are impacting the way aerial data has traditionally been collected and opening opportunities for smallscale high-density data acquisition. 'The multi-rotor drones capable to have higher payload that make it possible to carry heavy precise camera and even LiDAR sensor but less endurance. This causes the multi-rotor drones are generally utilised for very high accuracy mapping from small areas less than 100 hectares such as architectural 3D fine reconstruction applications' (Saadatseresht, et al. 2015), with this in mind, the suitability for use in open cut mining operations becomes clear with most aerial surveying conducted onsite focused around the extraction phase. In the case of rehabilitation, earthworks are confined to smaller areas due to haulage capabilities, smallscale high-resolution UAVs are a useful option for surveyors.

'UAVs are becoming more common place in a surveyor's tool kit especially low cost multirotor which can be bought straight off the shelf with little to no experience required to operate. UAV photogrammetry is a faster method for making higher quality, more reliable, with greater applications of products with legal validity, it costs several times less than field surveying due to its process automation without any huge field workings (Saadatseresht, et al. 2015). Unlike manned aerial surveys, UAVs enable the surveyor more flexibility with data collection and provide high quality data sets that are cost effective. The main disadvantage of aerial manned platforms such as airplanes is being expensive, especially for small study areas. During the last decades, low-cost Unmanned Aerial Vehicles (UAVs) are used to pass this handicap (Polat and Uysal 2017).

For use in mining where there is a real risk to personal safety while surveying around surface mining equipment (SME), traditional survey applications can put surveyors in 'the line of fire' whilst also being time consuming. Topographic survey using conventional equipment is proven to be extremely time consuming to take enough samples manually, and the execution is highly dependent on professionals ( $\mathrm{Du}$, et al. 2017). Through the reduction in field work and increased safety, it is easy to understand why UAV usage has increased significantly over the past decade.

Photogrammetry data has multiple applications in mining, with technical staff relying more and more on 3D digital models for planning, design checks and ground monitoring whilst having the luxury of high-resolution aerial images available, UAV is increasing becoming an invaluable resource. Three dimensional models are used as a planning and optimisation tool, to perform volume calculations, checking finished surface levels, and as built surfaces with UAV and TLS data making up most data collected and used in an open cut mining and rehabilitations works. Where time restraints allow UAV is fast becoming the preferred option for large pit surveys and inspections of vital equipment such as pipelines and electricity networks. This makes UAVs a versatile piece of equipment, '...comparing with traditional manned airborne platforms, they reduce the working costs and minimise the danger of reaching to risky study sites, with sufficient accuracy' (Polat and Uysal 2017). UAV applications are by no means limited to the production of surface models, however, can also be used for aerial asset monitoring if and when required. It can be stated that the UAV Photogrammetry can be used in engineering applications with the advantages of low-cost, time conservation, minimum field work, and competence accuracy. Moreover, the created 3D model is satisfactory to realise topography with texture (Polat and Uysal 2017).

\subsection{Combining datasets from different sources}

With multiple data acquisition techniques arise issues with how and why to combine spatial datasets, especially when these data sets can and most likely were intended to be used singularly. Combining spatial datasets is not a new concept, digital elevation models were being combined with conventional methods to create detailed mapping solutions before the rise of TLS and cost-effective UAV as discussed in Tilly, et al. (2016). Typical data combinations include GPS or Total station with LiDAR. The merging of data comes with challenges depending on the combination of sources. This is explored through previous studies where the limitations of small-scale data collection like GPS or Total Station to incorporate large scale collection from LiDAR. '...it is frequently advantageous to combine a total station and/or RTK survey with a LiDAR survey, to have excellent coverage of a floodplain but also accurate and precise subcanopy and channel bathymetric topography' (Scott, 2008). 
Further studies have shown significant improvements and advantages to the integration of spatial data sets to achieve fuller 3D surfaces and to eliminate data shadowing of both TLS and UAV point clouds such as Tong, et al. (2015); Tilly, et al. (2016); Sasak (2019); Wojcik et al. (2019); and Zang, et al. (2019). Some limitations to these studies were caused by weather conditions as in Sasak (2019), poor GPS signals in Cook (2017), and inconsistent terrain or vegetation for Flener, et al. (2013); Tilly, et al. (2016); and Akturk (2018).

It is important when combining UAV and TLS datasets, the validity of the acquisition can be established, and the accuracy of both sets is investigated. Methods on how to ascertain the accuracy of UAV data has been researched in Riveiro, et al. (2013); Ouedraogo, et al. (2014); Tong, et al. (2015); Tilly,et al. (2016); Jo and Kim (2017); Puniach and Kwartnik-Pruc (2018); Mora (2019); Sasak (2019). where the projects have made use of Structure-form-Motion (SfM) software programs. SfM has been established as a reliable processing software programs in previous studies Turner et al. (2012); Cryderman et al. (2014); Ouedraogo, et al. (2014); Cook (017). Structure-from-Motion or $\mathrm{SfM}$, requires only limited ground-control and is ideally suited to imagery obtained from low-cost, non-metric cameras acquired either at close-range or using aerial platforms (Javernick, et al. 2014).

The use of control points throughout UAV flights and TLS are also another common way to ensure the data collected meets the accuracy requirements of the study. Previous studies have used extensive check points and ground control to test the accuracy of the equipment and the collected data, these include Tong, et al. (2015); Tilly, et al. (2016); Du, et al. (2017). These check points were evaluated against the surface models to validate the appropriateness of the creation method, the variations between the check points and the surface arise due to the interpolation of the surface between points in the point cloud, as the check points do not form part of the surface model differences between check points and the surface almost always occur.

It is important when intending to combine datasets that the temporal constraints are considered. With mining there are rapid changes to a surface as material is being shifted minute by minute with a decision required on what collection method needs to be employed first. Ideally both the TLS and the UAV acquisition would be collected simultaneously in Jaud, et al. (2016), however, this is often not the case, especially among agricultural applications explored in Ouédraogo, et al. (2014). Assessment of the accuracy in these cases would require more manual registration and filtering of point clouds. There is a difference between filtering and reduction of point cloud data. Removing non-terrain objects to get bare earth is called filtering. The ground filtering is an essential step to separate points which are from the ground surface and which are from non-ground features for almost all topographic applications as with Polat and Uysal (2017); Whereas a reduction means there is a reduction in the density of the point cloud, sometimes these terms are used interchangeable, however, this project aims to reduce data and document the effects using 3D surface models and volume calculations.

Iterative closest point (ICP) algorithm for point cloud registration is based on an iterative process, it is slow at finding corresponding points between two-point clouds and is less efficient when registering large-scale, high-density point cloud scenes (Cheng etal, 2018). The ICP algorithm also requires high point cloud density; when this is low, registration errors may occur in the search for the closest point. In addition, time complexity is generally high with the ICP algorithm.

Research indicates good outcomes from the combination of datasets with studies pointing to using the laser scans as the primary source as it has already established reliability over many years of use, with the advantage of being an active system of acquiring data when compared to the passive systems used in UAV photogrammetry. Accurate results can be obtainable when combining datasets, this is especially the case for merging TLS and UAV, however, there is a need to plan data acquisition carefully with the aim of capturing the data within the same time period. In some cases, this can be difficult due to the fastchanging weather conditions, the topographical location including terrain and difficulty with access especially for TLS equipment.

Research method focused on validating one type of data against another, and the studies where combined UAV and TLS data were used to track changes of topography over time were successful. The studies which included volume calculations focused on validating the UAV data without merging of different collection methods.

It is plausible through merging UAV point clouds with laser scanned point clouds more accurate surface models can be created and utilised for volume calculations with the potential to improve reporting of stock volumes and influence mine planning operations. Volumes of topsoil stockpiles are of significance when planning rehabilitation operations as the area has a limited supply and there is a requirement to comply with the formal rehabilitation plan lodged with the government department for mining in Victoria.

\subsection{D surface models}

Accurate and more reliable topographical representation in the form of 3D surface models can be created using one or multiple forms of data collection method such as LiDAR, UAV and Terrestrial Laser Scanner The requirements of surface models can vary from erosion surface monitoring, vegetation growth monitoring for agriculture to stockpile monitoring for bulk materials, in the instance of mining. These models are a visual representation of the existing and changing surfaces over time and are commonly used to calculate volumes between the different variations of the surface.

With multiple data acquisition techniques arise issues with how and why to combine datasets, especially when these data sets can and most likely were intended to be used singularly. Combining datasets is not a new concept, digital elevation models were being combined with conventional methods to create detailed mapping solutions before the rise of TLS and cost-effective UAV. Typical data combinations include GPS or Total station with LiDAR. The merging of data comes with challenges depending on the combination of sources, this is explored through previous studies with the limitations of small scale data collection like GPS or Total Station to incorporate large scale collection from LiDAR '...it is frequently advantageous to combine a total station and/or RTK survey with a LiDAR survey, so as to have excellent coverage of a floodplain but also accurate and precise subcanopy and channel bathymetric topography' (Scott, 2008), Further studies have shown significant improvements and advantages to the merging of data sets to achieve fuller 3D surfaces and to eliminate data shadowing of both TLS and UAV point clouds as investigated 
in Tong, et al. (2015); Tilly, et al. (2016); Sasak (2019); Wojcik al. (2019); Zang, et al. (2019).

\section{MATERIAL AND METHODS}

\subsection{Study area}

The study area is a rehabilitation mine site which is located at the rural town Ouyen North West Victoria, Australia, as illustrated in the Figure 1. The site forms part of Iluka Resources Murray Basin operations. Woornack, Rownack and Pirro (WRP) was the site for a significant open cut mineral sands mine comprising of multiple open cut pits, the main pit reached a length of just over fifteen kilometres. Iluka Resources extracted mineral sands from the site, including zircon, rutile and ilmenites from 2012 to 2015 with the site now in the rehabilitation phase with a commitment to reconcile the disturbance of land and return productive lots back to farmers for agricultural production and re-establish crown land native vegetation reserves.

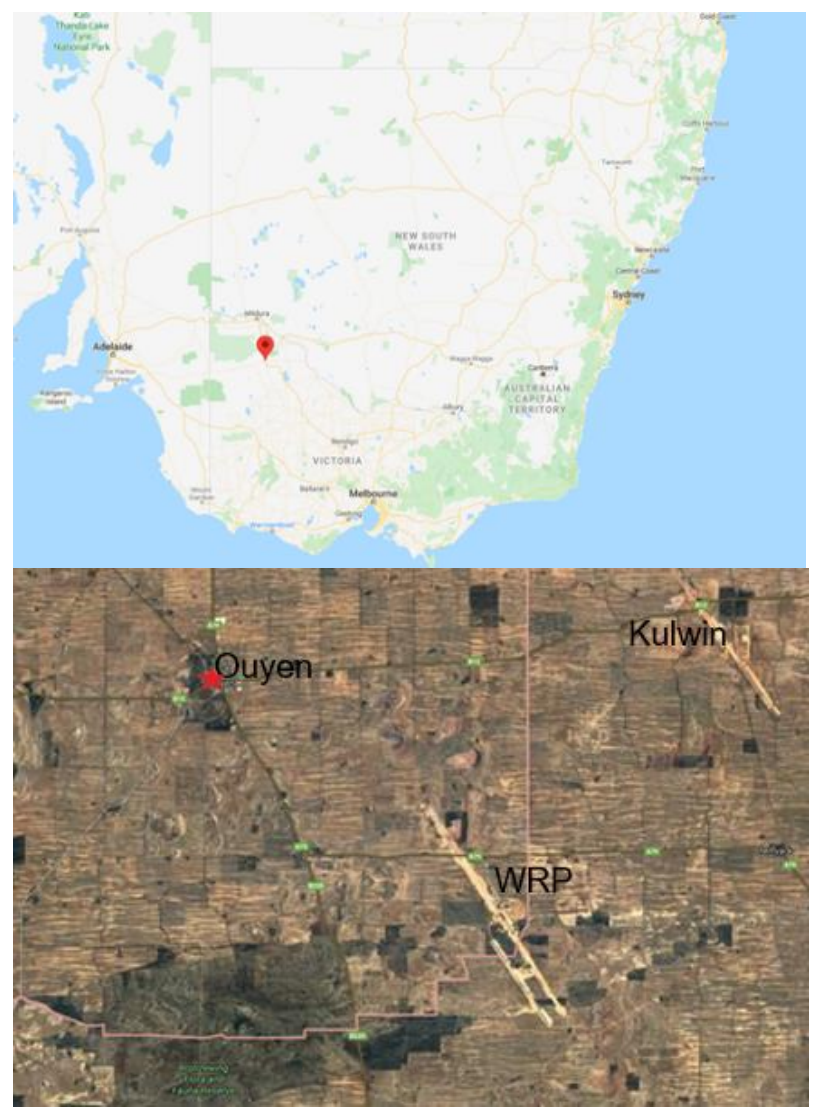

Figure 1: Map displaying the location of the Iluka Resources WRP Mine.

Two sites were selected for this study. Site 1 was chosen due to the flat consistent surface and offers little variation that could affect the outcome. The stockpile had recently been distributed over this site and the base was levelled to the subsoil design surface, with some modifications to suit the natural surface adjacent. The grid is measured roughly via the vehicle's odometer at $100 \mathrm{~m}$ spacings, the spacing is also adjusted to suit the topography as elevated ground is preferred for scanning stations. Area 1 was surveyed on the 16th of April 2020.

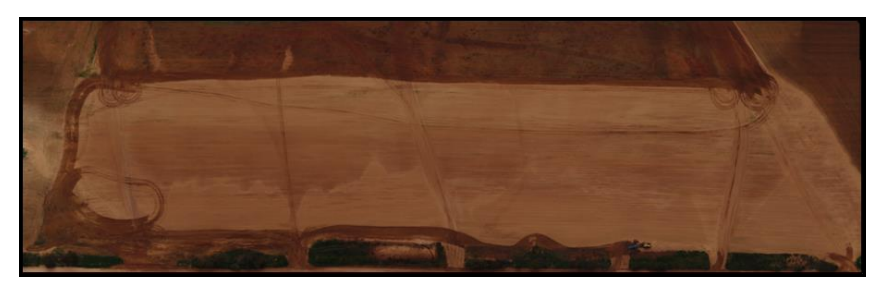

Figure 2: Aerial image of Site 1, the base of a subsoil stockpile at WRP Mine site.

Site 2 consists of a cluster of topsoil stockpiles that were cleared of vegetation along the top surface as highlighted in the Figure 3. The area was chosen as a typical scenario onsite as it was common practice for stockpiles to be placed in proximity. There are issues with this type of formation depending on the size and shape of stockpiles, in terms of laser scanning activities access and line of sight for the lasers can be difficult especially in the gullies between piles. To accurately scan these stockpiles multiple scanning stations is necessary, scan can be affected by the height of stockpiles and driveable access, leaving opportunities for gaps to exist within scans. These stockpiles cannot usually be scanned in a grid formation and scanning stations are dependent on the topography. Site 2 was surveyed on the 3rd of March 2020.

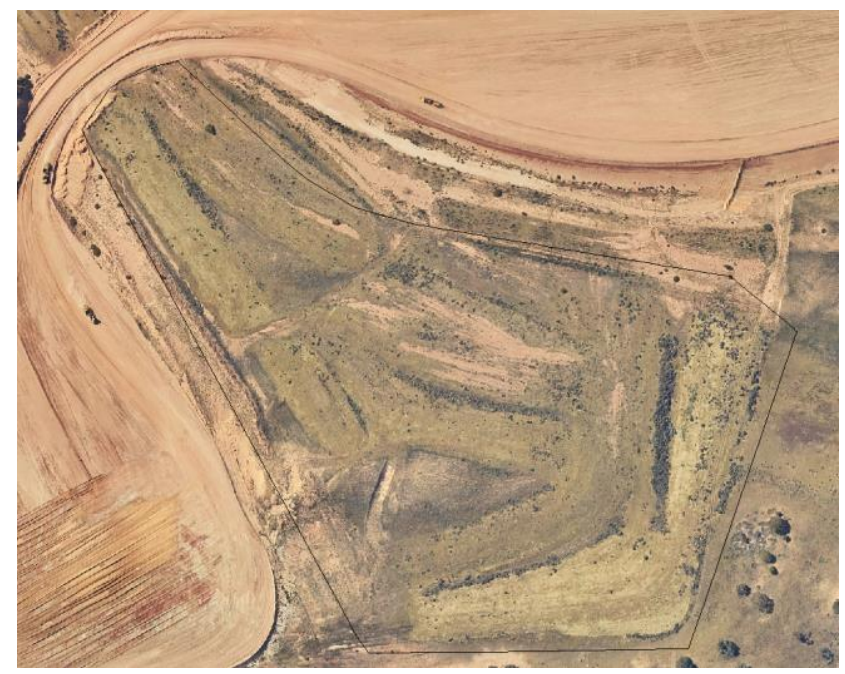

Figure 3: Aerial image of Site 2, a cluster of topsoil stockpiles at WRP Mine site.

\subsection{Data collection}

Field data were collected using UAV photogrammetry and Terrestrial Laser Scanning on two separate sites and each site was surveyed at different times. For ground control points, GNSS RTK Trimble R8 Rover connected through radio link to a fixed base station on the Iluka Resources WRP Rehabilitation site was used. Coordinates captured as TOPO points with a 5 second, 3 EPOCHS read for each ground control point (GCP) and scanning station, including backsight. During the field data collection, Iluka Resources WRP mine sites rules and regulations were followed which includes site inductions, traffic management procedures, safety policies and procedures and task specific Safe Work Instructions (SWI). All surveying onsite adhered with the WRP Survey Procedure and UAV data collection SWI - Use of RPA (Remotely Piloted Aircraft) on Rehabilitation Sites. 


\subsubsection{Terrestrial laser scanning data capture}

Terrestrial laser scanning was conducted using a Maptek 8820 terrestrial laser scanner mounted to the roof of a Toyota Hilux 4WD via the manufacturer's vehicle mount bracket and vehicle roof racks. The roof racks and the mount were fitted to the vehicle and are periodically checked for levels by the Iluka technical staff to ensure set up quality is maintained. The 8820laser scanner has been used at the WRP site for over 4 years and has become a valuable tool in capturing topographic data. When scanning was conducted, the scanner was set up and mounted on the vehicle. All scanning was conducted with the operator inside the vehicle for maximum safety which was an onsite health and safety requirement.

As part of the scanner set up, a Trimble R8s GNSS rover was connected to the top of the scanner unit via a thread on the scanning unit and a $135 \mathrm{~mm}$ extension pole, the GNSS rover was controlled using a Trimble TSC 3 and coordinate points were recorded using the onsite RTK network.

Scanning positions were determined by the operator and based on the topography of the target surface, the heavy machine activities and personal experience from using the equipment. The aim was to scan the stockpiles to include as much of the topography as possible so a complete surface could be extracted from the scan point clouds with as little data shadows and voids as possible. When scanning stockpiles, the geometry of the target surface is challenging, this can result in data shadows as the line of site to the surface can be obstructed by the geometry of the stockpile itself or the limitations from the scanning angle based on the laser scanners specifications.

The top surface of the stockpile was cleared of vegetation with heavy machinery, grader or dozer prior to scanning, however, the sides of the stockpile remain vegetated to ensure wind erosion was kept to a minimum. The vegetation on the side of stockpiles consists of native grasses and introduced weeds and could be filtered out of the scans during registration and processing.

To use the scanner, the operator drove to the first scan point, inserted the scan parameters in the Panasonic Toughbook tablet using the Maptek scanning software, numbers the stations from 100 and creates a backsight of 101, conducts the scan and stores a TOPO point using the GNSS rover and data logger starting at 100. After the 360-degree scan is completed the operator will reverse the vehicle approximately $10-15 \mathrm{~m}$ and store a second point, 101, for the backsight. By setting up and storing a backsight in the field, scans can be registered against these points when processing in the i-Site software. The operator proceeds to move and scan at various scanning stations until satisfied they had enough data. Area 1 consists of fourteen laser scanning stations over 8.4Hawith the scanning station on a grid of approximately $100 \mathrm{~m} \times 100 \mathrm{~m}$. Area 2 was covered in fourteen scans over 9.7ha, this required close scans taken from on top of stockpiles and around the perimeter where accessible by vehicle.

\subsubsection{UAV data capture}

On the same day laser scanning was conducted, the area was flown with a DJI Matrice 210 UAV fitted with a survey grade camera, Zenmuse X4S and a Loki PPK unit.

It was important to conduct the scans and the UAV flights on the same day to minimise the differences between the collected surface data. This was to ensure material movement on the stockpiles is kept to a minimum, and in this case, there was no movement of material from the stockpiles by surface mining equipment between each data acquisition. Flights over stockpiles were conducted as part of larger site wide surveys.

Ground control points (GCP) were established using the local RTK network and the GNSS Trimble rover and TSC3. Targets were painted with white line marking paint onto the ground in a grid formation over the target site, the targets were $1 \mathrm{~m}$ in diameter with a "Y" target for the GCP tie points and an "X" for the control points, these were large enough to see in photographs when processing. Targets were set out in areas where there was little chance of disturbance from mining operations and machinery, however, as this is a common occurrence, extra control points were set out for redundancy. After ground control was in place, a temporary base station was established over a known marker. A static survey was started to $\log$ a position at 1 second intervals as required for the PPK Loki system.

With both ground control stations, and a logging survey established UAV flights were conducted using the DJI GSP pro application on an iPad. The settings for both flight areas had the same settings including flight height, speed and side and front overlap to keep consistency across the study.

Flights for Area 1 were conducted from 10:00am on the 19th of April 2020, during good weather conditions with winds well below the $40 \mathrm{~km} /$ hour maximum of the Matrice 210's capability. Flights for Area 2 were conducted on the $3^{\text {rd }}$ of March between 2:00pm and 4:00pm. For both occasions' flights were scheduled around active earthworks due to CASA regulations and site restrictions relating to the proximity of UAVs to people and the inability to fly within $15 \mathrm{~m}$ of any individual. Take-off and landing sites were chosen so as not to interfere with earthworks operations and within the flight zone to minimise UAV travel to and from the 'Home' point.

Once an appropriate "Home" location was set, the operator assembled the UAV and completed the necessary pre-flight checks to ensure the safety and success of the flights. Using the radio controller in programmed $(\mathrm{P})$ mode, the DJI Go fly app was used to ensure camera settings were satisfactory for current conditions. Once all the pre-flight checks and settings were confirmed, flights were commenced and completed.

\subsection{Data processing}

Mining companies have preferred software for data processing, and although onsite UAV operations in mining is relatively new, the most popular software for creating photogrammetric outcomes such as point clouds, and orthomosaics is Pix4D mapper.

For general survey data processing, the equipment types and brand dictate the processing software, for this project Maptek scanner and associated software was used for the terrestrial laser scanned data and the LAS file generated through Pix4D. For data analysis, Surpac, a popular software package for mining surveyors, engineers and geologists was used for some data representations and volume generation.

Each data set requires initial processing through brand specific software. For the survey stations of the laser scanner and the ground control, the Trimble .job file was processed through Trimble Business Center to export a csv point file for use in Maptek i-Site (survey stations and backsights) and Pix4D Mapper (Ground Control Stations). 
The Loki PPK data was processed through ASP Suite, this is a brand specific processing software that is required by GEOCUE, the manufacturer of Loki. ASP Suite uses data from the UAV in the form of .DAT files combined with the GNSS logging file converted from a .T02 file converted through RINEX and the logging files from the Loki system. Combining the 3 data sources the software geotagged the photographs ready for processing through Pix $4 \mathrm{D}$.

All UAV data was processed using commercially available Pix4D software. Pix4D creates Structure Form Motion (SfM) point clouds which have been tested for accuracy through previous studies, as with Turner, et al. (2012). Pix4D is a reliable software package for photogrammetry processing and isn't directly associated with a brand of UAV or camera. Images were processed using the 3D maps option in Pix4D Mapper, which created. LAS DTM file and an orthomosaic image. Accuracy of the processing was assessed checking the generated report after initial processing. The report contained information relating to the accuracy of the images based on the GCPs and specified control points. Using geotagged images is a slightly different process and requires less GCPs. The excess GCPs were used as control check points to compare against the final generated data.

Maptek i-Site, a 3D modelling program was used to process the terrestrial laser scan point cloud data. Each scan was assigned a point ID and a backsight ID during the data collection. Together with the point file the scans were registered within the software, the global positioning function was then used to fine-tune the registration of scans. Scans were then filtered first by range, 10$250 \mathrm{~m}$, this was to eliminate the vehicle from the scans and any points outside of $250 \mathrm{~m}$. In addition to this, this also assisted in the registration process. Global registration was reapplied until the scans reached their best registration results, once this occurred the function Smart Connect was used to connect like points within the scan based on point can cell geometry, and global positioning could be used to further enhance the scan registration. When the scans reach registration better than $0.50 \mathrm{~m}$, scan could be separated. Once satisfied the scans had reached optimal registration, filtering of points occurred on that process.

Filtering Point clouds is a major step to prepare for the merging of datasets. Laser scanned points were filtered to $0.2 \mathrm{~m}$, this allowed for quicker and smoother processing on the computer network. At this stage, scans were assessed for data shadows and holes that might have affected the surface model and the volumes.

The LAS file created from Pix4D was imported into i-Site for further processing. The files were large files and for ease of use were filtered and reduced through this software, a degree of filtering had already taken place in the generation of data through Pix4D. Vegetation is one of the features that was filtered out . The option to filter out vegetation before importing into i-Site made the process of filtering more consistent. The target area was isolated through clipping the point cloud, this reduced the number of points and increased the processing speed. The LAS file was first filtered to $0.2 \mathrm{~m}$ and using the laser scan data as the base object, the LAS file is filter using a proximity filter to $0.2 \mathrm{~m}$.

After each dataset was processed and filtered the surface model was created. By selecting the scans and/or LAS files together the Topographic Surface function in i-Site was used to create one model. The models created were a TLS data model, a UAV data model and a combined data model. The models were 'despiked' to remove outlying spikes and points and assessed for irregularities. When satisfied with the quality of the model, fish net grid was produced over the surfaces at 1-meter spacings. Each fishnet grid shared the same $(\mathrm{X}, \mathrm{Y})$ coordinates however the $(\mathrm{Z})$ values depended on the elevation of the surface models.

\section{RESULTS}

\subsection{Model to model comparison}

The target areas as illustrated in the Figure 4 shows significant differences between the TLS and UAV surface models when compared on a fishnet grid of $1 \mathrm{~m} \times 1 \mathrm{~m}$. The variance was filtered to $+/-50 \mathrm{~mm}$ to identify outliers. The tolerance of $50 \mathrm{~mm}$ was established due to the type of material and the tolerance used during earthworks.

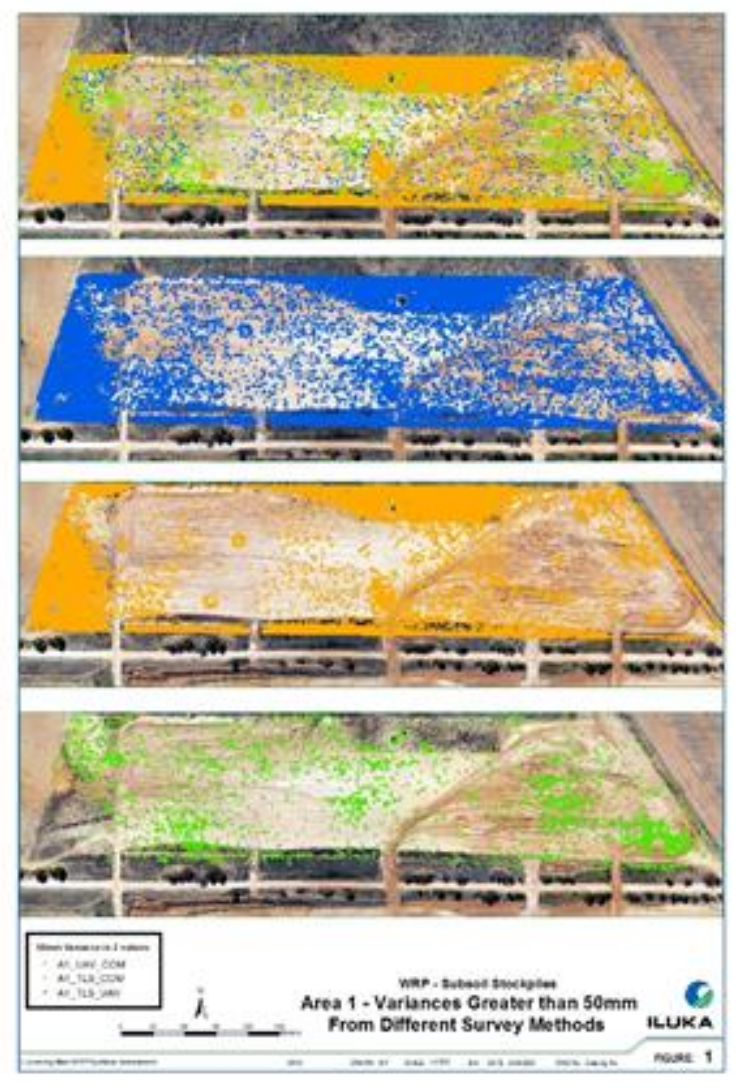

Figure 4: Map of Area 1 indicating the $+/-0.05 \mathrm{~m}$ variations between the TLS, UAV and Combined Surface Models.

The above variance can be attributed to the topography of the area with laser scans containing data shadows. It is easy to identify the areas devoid of scanned points in the software and to cross reference against the variation model. Some attributes that also lead to data shadows and void points are wheel tracks due to soft ground that get missed in scanning operations and vegetation that is difficult to filter out without over filtering of the surface.

As illustrated in the Figure 5, Area 2 has issues with gullies between stockpiles, when the stockpiles are so close, vehicle 
mounted laser scanning limits the station options available. The gullies between stockpiles show up as holes in the data and this is one of the reasons a secondary survey with UAV is conducted. The blue points indicate the difference between UAV and TLS collection, it is easy to see where the laser scans have missed data due to the scan positions and the topography. When the TLS and UAV point clouds are combined to create a new surface model, there is a reduction in the number of points outside the $50 \mathrm{~mm}$ tolerance.



Figure 5: Map of Area 2 indicating the $+/-0.05 \mathrm{~m}$ variations between the TLS, UAV and Combined Surface Models.

As in previous studies there is a visual assessment to identify areas where vegetation or line of sight from the TLS could not be established. 'A visual assessment of high vertical difference against the orthorectified imagery shows that error is particularly pronounced around trees, and in areas vegetated with heather... Dense vegetation types obstruct line-of-sight of actual ground level, thus generate a vertical difference between the two datasets' (Tonkin et al., 2014).

\subsection{Volume calculations}

Volume calculations were performed against the starting surface of the stockpile, the start surface was created when the stockpile was first established and was surveyed via vehicle continuous TOPO with a GNSS rover mounted to a vehicle. Table 1 shows the volume differences between each version of the stockpile surface model for both Area 1 and Area 2.

The differences across Area 1 were minimal, and this could be attributed to the flatness of the stockpile, as the base of the stockpile was shaped to the finished design surface, the variation across the surface was minimal. Area 2 contains greater variations between the TLS surface when compared to the UAV surface and the combined surface. This is most likely attributed to the data shadows and holes within the laser scans discussed in above.

\begin{tabular}{|c|c|c|}
\hline \multicolumn{3}{|c|}{ Area 1} \\
\hline \multirow[t]{2}{*}{ Surface } & \multicolumn{2}{|c|}{ Volumes Between Surfaces } \\
\hline & Cut Vol m3 & Fill Vol m3 \\
\hline TLS Surface & 182 & 140238 \\
\hline UAV Surface & 151 & 139812 \\
\hline Combined Surface & 163 & 140080 \\
\hline $\begin{array}{ll}\text { Difference } & \text { TSL/ } \\
\text { Combined } & \end{array}$ & 19 & 158 \\
\hline $\begin{array}{l}\text { Difference } \\
\text { Combined }\end{array}$ & -12 & -268 \\
\hline \multicolumn{3}{|c|}{ Area 2} \\
\hline \multirow[t]{2}{*}{ Surface } & \multicolumn{2}{|c|}{ Volumes Between Surfaces } \\
\hline & Cut Vol m3 & Fill Vol m3 \\
\hline TLS Surface & 3478 & 94 \\
\hline UAV Surface & 512 & 561 \\
\hline Combined Surface & 470 & 1471 \\
\hline $\begin{array}{ll}\text { Difference } & \text { TSL/ } \\
\text { Combined } & \end{array}$ & 3008 & -1277 \\
\hline $\begin{array}{l}\text { Difference } \\
\text { Combined }\end{array}$ & 42 & -910 \\
\hline
\end{tabular}

Table 1: Surface volume calculations between start stockpile surface and new surface models.

As a comparison, the historical topsoil model was compared against the TLS, UAV and the combined models. There was a $25 \%$ difference between the historical model and the TLS model, $19 \%$ difference to the UAV model and $20 \%$ difference in the combined model. On a stockpile of this size, this equates to 14627, 10971, and 11847 bulk cubic meters (BCM) respectively. This is of significant value in terms of topsoil placement for future agricultural productivity. If the topsoil thickness were to be laid out at $50 \mathrm{~mm}$ the area of coverage ranges from $590 \mathrm{~m}^{2}$ to $731 \mathrm{~m}^{2}$ and an estimated haulage cost of between $\$ 3000$ and $\$ 4500$ at current rates.

\section{DISCUSSION}

In this study the creation of surface models using data sets derived from different survey methods, TLS and UAV, was investigated in terms of fit for purpose use and accuracy. The main objectives were (i) Investigate the accuracies of both laser scanning and low-cost UAVs in the creation of surface models.

(ii) Combine data sets and test the volumetric results against terrestrial laser surfaces, photogrammetry (UAV) surfaces and combination surfaces, (iii) Perform a cost analysis based on the various collection and data types in terms of bulk cubic meter rates, and (iv) Determine a best practice guideline for combining of data sets for open pit mining operations.

Combing UAV and TLS data for the purpose of mining use can be an acceptable method of acquiring large datasets with minimal data shadowing or data holes and providing the accuracy requirements can be established and met. For this study the TLS was the premier standard of data collection for the rehabilitation operations and the UAV was required to meet these accuracy standards. Through the processing and 
comparison of data sets the UAV data performed very well against the TLS with variations between surfaces able to be identified.

The current methods of data collection on the study site is the continuous TOPO used in cases where TLS cannot be utilised. It's not often the terrestrial laser scanner isn't available, only with calibration and vehicle unavailability likely the cause. Historically data from around site was collected only through GNSS rover with LiDAR periodically acquired but not used for the calculations of material volume balance. There has been opportunity with technology adoptions to improve the survey processes to include larger data gathering and methods. The mining software Surpac has been utilised over the duration of the mine with most survey data converted to this format. By utilising this software older pre mine and start of mine surfaces are available in the same format currently used, allowing for surface comparison without conversions of data.

Merging of survey methods and data sets is a valid option for surveyors, especially terrestrial laser scanning and UAV photogrammetry, this is discussed in Riveiro, et al. (2013); Polat and Uysal (2017); and Sasak (2019). These methods complement each other, and both have strengths and weaknesses. 'The general advantage of laser scanning over photogrammetry is in the ability of sampling serval kinds of surfaces (e.g. Top of vegetation canopy, inter-canopy surfaces and ground) which are in the line of sight of the laser beam until impermeable surface restrains further penetration of the laser energy' (Sasak, 2019). One of the strengths of laser scanning is the quality of scans for flat uniformed surfaces when compared to the UAV which struggles with lack of variation in the surface and high reflectance. For this study, flat surfaces were an issue due to the recent earthworks in the area and moisture in the soil. However, if the surveys were conducted on weathered smooth stockpiles, the surface could have played a role in data shadows and void points. A weakness of terrestrial laser scanning that is a strength of UAV surveying is requirement of line of sight. Line of sight can be an issue where vegetation and topography interfere with the laser signal. A minor issue during this study was surveying close to stockpiles where there is no undercut of highwalls or tree canopies, UAV coverage can infill where the scanner has missed.

Filtering of data has the potential to affect the outcomes of merging datasets. There are indications the data used as the primary source could influence the results, and it is up to the surveyor to make judgement on how to prioritise the collection and use of datasets. There is a requirement when performing the collection surveys to be aware of how the collection method is going to change or influence the results.

There are some limitations to the research which include the size and the type of areas chosen for the study. The area although different were used due to the accessibility and thorough knowledge of the site. Both areas were under 10ha with similar conditions. Ideally more stockpiles could have been used to compare the results. Larger more diverse surfaces could have influenced the results. The age of equipment could also factor into the results, with the scanner being over 4 years old and subsequent models have been produced, the Trimble receiver was of an earlier generation and unable to receive correction from the newer satellite constellations. The UAV was the newest piece of equipment, with less than 5 flying hours at the time of surveys, more experimentation on flight settings could have taken place to ascertain the best settings for the task.

\section{CONCLUSIONS}

The purpose of this research paper was to determine if combining the data from UAV and TLS could be effective in the creation of $3 \mathrm{D}$ surface models to be used in volumetric computation for the mining industry. With a focus on rehabilitation operations and restoring the land surface for farming and native vegetation.

This research leveraged on the survey methods used in the collection of data based upon similar studies outlined in the literature review. The literature review findings reveal the extent if data merging within the spatial science fields but also the agricultural and environmental science applications. Various methods are employed based on the type of collection and the environmental factors such as terrain and vegetation. LiDAR is commonly combined with total stations and GNSS to improve the quality of elevations in both agricultural and environmental applications.

Increasingly TLS and UAV are being utilised for forestry mapping and land surface monitoring in natural environments and are being combined with sonar system when mapping of waterways is required. Mining deals with many surface changes, with earthworks sculpting new surfaces in a planned and predictable manner. The planning and execution of mine surveys has more overall control than say land slide monitoring, which is one of the advantages of this study.

It is possible to successfully combine multiple datasets from different surveying techniques, though it is important to assign a primary source of data based on the task and the judgement of the surveyor and what accuracies are required. This sentiment is echoed through Sasak (2019) who used the TSL data under the assumption of greater accuracy due to the referencing process. Thorough examination and analysis of the results is also advised to ensure the quality of the results. The individual accuracies of each component were not calculated as part of this research. This is due to the accepted accuracy of the equipment used on these sites with the TLS proven results overtime. For the UAV, previous flights had been registered and checked against scans and the errors were found to be acceptable and traceable to events or known issues with the collection method. In the past and periodically throughout the year onsite data is checked against GNSS continuous TOPO surveys and assessed for accuracy with equipment calibrations performed as required.

There is scope for further testing especially for larger areas and varying ground conditions. Investigating uniform or surfaces of high reluctance and how to minimise the effects of these conditions could be of use in a mining context. It is common for surfaces to be striped and weathered over time with surveyors required to do multiple data collections before there is a change in conditions. With areas large enough to make TLS time consuming and work intensive, UAV could present a more viable option, with TLS to fill in the gaps of data when required. The research confirms the possibilities of combining the active laser data with the passive UAV data effectively to achieve good results in the creation of surface models quickly and accurately enough to use in earthwork estimation and volume calculations.

The volume calculation difference between the surfaces of both subject areas were minimal, with the biggest variation in volumes traceable to data shadowing in the TLS data in Area 2. The volume difference of approximately $3000 \mathrm{~m} 3$ was not significant enough to cause alarm, especially when upon 
investigation into the data the cause could easily be identified. As the sight was a small area the ability to review and identify these errors was straightforward. If the area were of a sizable amount, closer to the 100ha limits of UAV, identifying inconsistencies would have taken more effort and the point cloud registration would have used ICP algorithm.

Although best practice guidelines were not created as part of this research, it is possible the outcomes can contribute to their creation. For the specific operations and site conditions relating to the site, it is plausible that a procedure could be created and adopted as part of the company's survey operations. Results are encouraging for similar operations working with earthworks and stockpiled materials. However, the use of both methods of data collection is more suited to large areas where time restrictions for accurate data are critical, and one method (TLS) would take longer than employing two (TLS and UAV). This study has not used ICP algorithm during point clouds registration and it is recommended for a further study to use this algorithm to improve the quality.

\section{ACKNOWLEDGEMENTS}

We would like to thank Iluka Resources, in particular, the Eastern Australia Rehabilitation Manager and the WRP/ Kulwin Site Coordinator for their assistance during field works and allowing us to use equipment and resources to complete this research project.

\section{REFERENCES}

Akturk, E., Arif, A. (2018). "Accuracy Assessment of a LowCost UAV Derived Digital Elevation Model (DEM) in a Highly Broken and Vegetated Terrain." Measurement 136: 382-386.

Cheng L., Chen S., Liu X., Xu H., Wu Y., Li M., and Chen Y. (2018). Registration of Laser Scanning Point Clouds: A Review. Sensors. 2018;18:1641. doi: 10.3390/s18051641

Cook, K. L. (2017). "An Evaluation of the Effectiveness of Low-cost UAVs and Structure from Motion for Geomorphic Change Detection." Geomorphology 278: 195-208.

Cryderman, C., et al. (2014). "Evaluation of UAV Photogrammetric Accuracy for Mapping and Earthworks Computations." Geomatica 68(4): 309-317.

Du, M., et al. (2017). "Topographic Mapping of Farmland by Integration of Multiple Sensors on Board Low-Altitude Unmanned Aerial System." World Academy of Science, Engineering and Technology International Journal of Computer and Systems Engineering: 1131-1135.

Flener, C., et al. (2013). "Seamless Mapping of River Channels at High Resolution Using Mobile LiDAR and UAVPhotograph." Remote Sensing 5: 6382-6407.

Iluka Resources. (2009) "Mineral Sands Technical Information" [Online].Available:

https://www.iluka.com/getmedia/67904b40-4eae-4624-8a16720f4874244a/iluka_technical_brochure_07_12_09.aspx (Accessed 02 February, 2021).

James, L. A., et al. (2012). "Geomorphic Change Detection using Historic Maps and DEM Differencing: The Temporal
Dimension of Geospatial Analysis." Geomorphology 137(1): 181-198.

Jaud, M., et al. (2016). "Assessing the Accuracy of HighResolution Digital Surface Models Computed by PhotoScan and MicMac in Sub-Optimal Survey Conditions." Remote Sensing $8(6)$.

Javernick, L., et al. (2014). "Modelling the Topography of Shallow Braided Rivers using Structure-from-Motion Photogrammetry." Geomorphology 213: 166-182.

Jo, Y. H., and J. Y. Kim (2017). "Three-Dimensional Digital Documentation of Heritage Sites Using Terrestrial Laser Scanning and Unmanned Aerial Vehicle Photogrammetry." ISPRS - International Archives of the Photogrammetry, Remote Sensing and Spatial Information Sciences XLII-2/W5: 395-398.

Mora, O. E., Suleiman, A., Chen, J., Pluta, D., Okubo, M. H., \& Josenhans, R. (2019). "Comparing sUAS PhotogrammetricallyDerived Point Clouds with GNSS Measurements and Terrestrial Laser Scanning for Topographic Mapping." Drones 3(3): 64.

Ouédraogo, M. M., et al. (2014). "The Evaluation of Unmanned Aerial System-based Photogrammetry and Terrestrial Laser Scanning to Generate DEMs of Agricultural Watersheds." Geomorphology 214: 339-355.

Polat, N. and M. Uysal (2017). "DTM Generation with UAV Based Photogrammetric Point Cloud." The International Archives of the Photogrammetry, Remote Sensing and Spatial Information Sciences, 77-79.

Puniach, E. and A. Kwartnik-Pruc (2018). "The Use of Laser Scanning and Unmanned Aerial Vehicles in Construction Surveying in the Light of Legal Regulations in Poland." Journal of Applied Engineering Sciences 8(2): 79-88.

Riveiro, B., et al. (2013). "Validation of Terrestrial Laser Scanning and Photogrammetry Techniques for the Measurement of Vertical under Clearance and Beam Geometry in Structural Inspection of Bridges." Measurement 46(1): 784-794.

Saadatseresht, M., et al. (2015). "UAV Photogrammetry: A Practical Solution for Challenging Mapping Projects " The International Archives of the Photogrammetry, Remote Sensing and Spatial Information Sciences, 619- 620.

Sasak, J., Gallay, M., Kanuk, J., Hofierka, J., \& Minar, J. (2019). "Combined Use of Terrestrial Laser Scanning and UAV Photogrammetry in Mapping Alpine Terrain." Remote Sensing 11(18).

Scott, J. A. (2008). Creating Digital Elevation Models from Combined Conventional and LiDAR Topographic Surveys. TS105.1.

Szentpeteri, K., et al. (2016). Drones (UAVs) in mining and Exploration. An Application Example: Pit Mapping and Geological Modelling, MGEI 8th Annual Convention, Indonesia, 5-6 October, 2016.

Tilly, N., et al. (2016). "Geomorphological Mapping with Terrestrial Laser Scanning and UAV-Based Imaging." ISPRS International Archives of the Photogrammetry, Remote Sensing and Spatial Information Sciences XLI-B5: 591-597. 
Tong, X., et al. (2015). "Integration of UAV-Based Photogrammetry and Terrestrial Laser Scanning for the ThreeDimensional Mapping and Monitoring of Open-Pit Mine Areas." Remote Sensing 7(6): 6635-6662.

Tonkin, T. N., et al. (2014). "The Potential of Small Unmanned Aircraft Systems and Structure-from-Motion for Topographic Surveys: A Test of Emerging Integrated Approaches at CWM Idwal, North Wales." Geomorphology 226: 35-43.

Turner, D., et al. (2012). "An Automated Technique for Generating Geo-rectified Mosaics from Ultra-High Resolution Unmanned Aerial Vehicle (UAV) Imagery, Based on Structure from Motion (SfM) Point Clouds." Remote Sensing 4(5): 13921410.

Victoria State Government. (2014) "Code of Practice for Mineral Exploration Standards, Procedures and Practical Guidance under the Mineral Resources (Sustainable Development) Act 1990." Department of Economic Development, Jobs, Transport and Resources (DEDJTR).

Victoria State Government (2020). Mineral Sands. [Online] Available

https://earthresources.vic.gov.au/_data/assets/pdf_file/0004/46

1758/Mineral-Sands-Factsheet-February-2019.pdf

(Accessed 2 February, 2021).

Victoria State Government. (2018). "Preparation of Work Plans and Work Plan Variations Guideline for Extractive Industry Projects". Department of Economic Development, Jobs, Transport and Resources (DEDJTR).

Victoria State Government (2018). "Earth Resources Regulation Compliance Strategy 2018-2020" Economic Development, Jobs, Transport and Resources, Earth Resources Regulations, Victoria Government.

Wojcik, A., et al. (2019). "The Use of TLS and UAV Methods for Measurement of the Repose Angle of Granular Materials in Terrain Conditions." Measurement 146: 780-791.

Zang, Y., et al. (2019). "An Accurate TLS and UAV Image Point Clouds Registration Method for Deformation Detection of Chaotic Hillside Areas." Remote Sensing 11(6). 